\title{
Design and Implementation of an IDC Environmental Monitoring Controller Based on Arduino
}

\author{
Xiao-Qiang Jia \\ Network Center of IMUT, Hohhot 010051 \\ Inner Mongolia, China \\ jiaxiaoqiang@imut.edu.cn
}

\author{
Gang Wang \\ Network Center of IMUT, Hohhot 010051 \\ Inner Mongolia, China \\ wg@imut.edu.cn
}

\begin{abstract}
In view of the real-time IDC environment monitoring, this paper designs a kind of environmental condition monitoring system based on the Arduino. The system takes the Arduino mage2560 and all kinds of sensors as the core, to design and write the Arduino mage2560. It realizes monitor the IDC environment changes of temperature, humidity, illumination and noise in real time and conveniently, and the sensors data is sent to the OneNET ioT platform. Practice shows that this design can realize data collection economically and efficiently, and can be used for fast real-time IDC environment monitoring.
\end{abstract}

Keywords-Arduino.Esp8266.OneNET.IDC environment monitoring

\section{INTRODUCTION}

With the rapid development of the Internet of things technology, the way of operation and maintenance management of IDC computer room also has a qualitative change. By the original tradition of relying solely on human, operation and maintenance personnel rely on the experience of operation and maintenance of the computer room operation and maintenance, emergency handling methods developed for the current power and environmental monitoring system by the intelligent power plant automatic operation and maintenance of the way. The project for the data center of our university in the operation and maintenance are encountered in the measurement of environmental parameters is not accurate is not comprehensive, not flexible configuration, alarm mode single, error alarm, and cannot be viewed on a mobile device, expensive, installation deployment of complex engineering status of. Design and development of a mage2560 Arduino[1] based on the measurement parameters comprehensive, flexible configuration, easy to install, support for Mobile Devices see IDC environmental parameters acquisition controller.

\section{SYSTEM COMPONENTS}

The main research goal of this paper is to design and develop a set of IDC based Arduino environment parameter acquisition controller as shown in Figure 1.

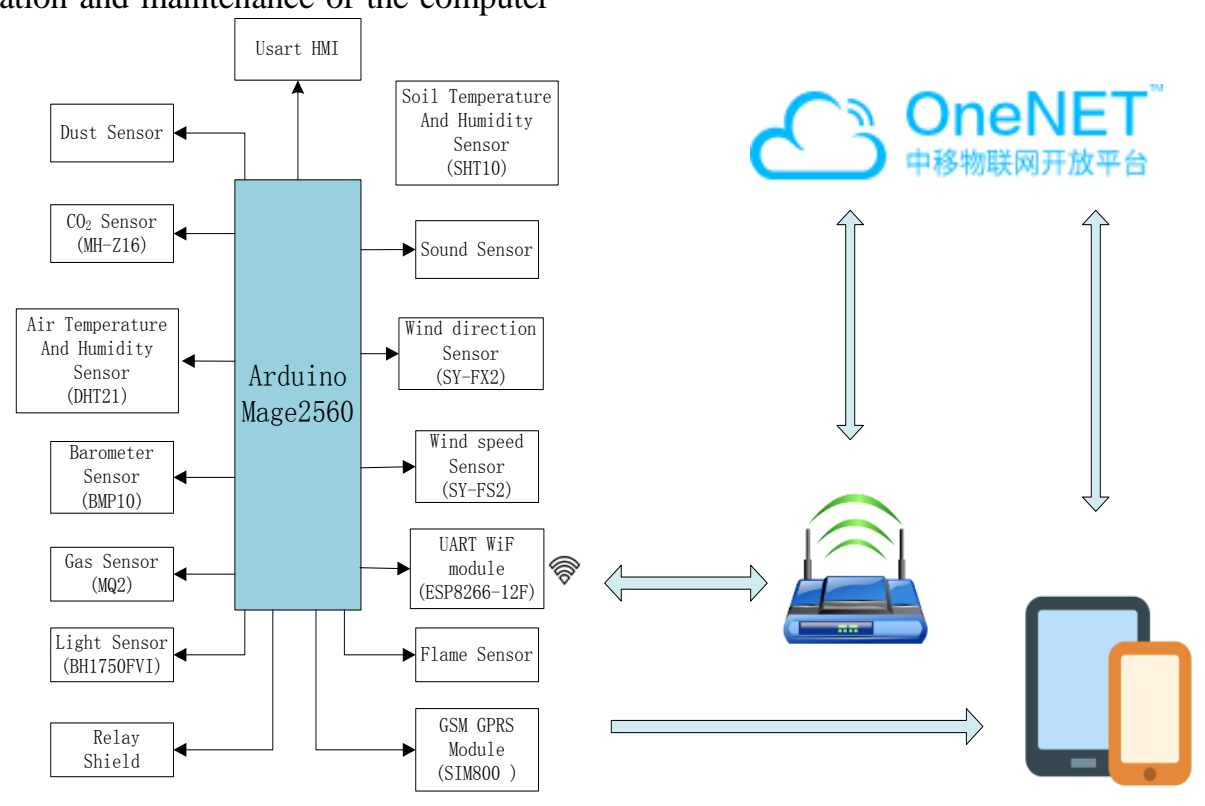



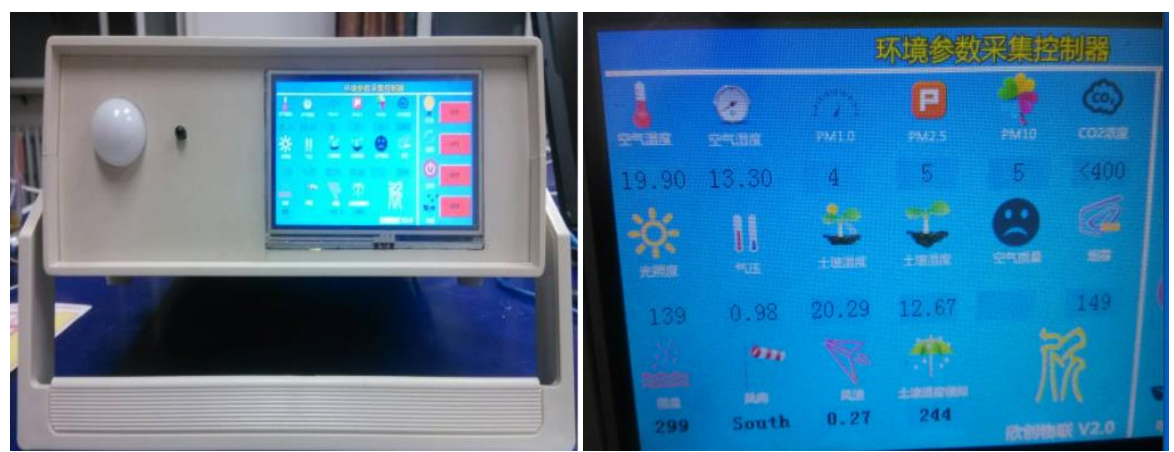

Figure 1. IDC environment parameter acquisition controller

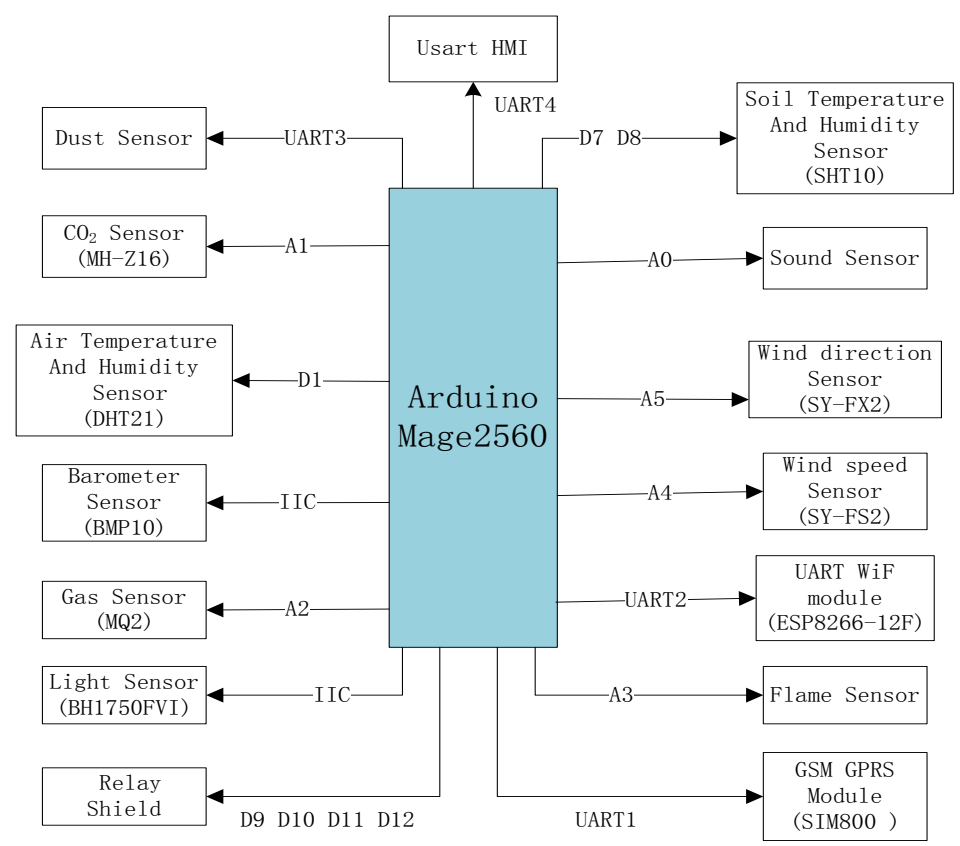

Figure 2. Schematic diagram of the hardware modules

Acquisition and control is first applied to our school environment parameters in virtualized data Virtual data center maintenance teacher can use mobile phone or tablet touch screen, Internet access equipment in the office or home remote telemetry to virtual data center air temperature, air humidity, dust, water immersion, smoke, flame, hydrogen, pue, the thunderstorm number, electromagnetic radiation, room noise environmental parameters and to measure the parameters through the dashboard, graphs, bar charts, pictures, text mode display. Users can freely configure the parameters display interface according to their favorite style or data. Can also be found that the room lights, doors and windows, shade and other state of the switch state. To facilitate the school virtual operation and maintenance teachers to understand the computer room environment parameters and running state, reduce the workload of inspection.

\section{A. The Composition of the Hardware System}

The Mega 2560 is a microcontroller board based on the ATmega2560. It has 54 digital input/output pins (of which
15 can be used as PWM outputs), 16 analog inputs, 4 UARTs (hardware serial ports), a $16 \mathrm{MHz}$ crystal oscillator, a USB connection, a power jack, an ICSP header, and a reset button.[1]As Figure 2 shown, Liquid crystal display screen connected with four serial Arduino mage2560, WiFi chip esp8266, dust sensor and GSM GPRS module; connected through the IIC bus and the air pressure sensor bmp10 and soil temperature and humidity sensor SHT11 and Air temperature, air humidity, dust, water leaching, smoke, flame, hydrogen, PUE value, the number of thunderstorms, electromagnetic radiation, noise and other sensors connected to digital or analog ports.

The system is based on the mage 2560 Arduino main control board, the use of mage $2560+$ esp8266 network to achieve the function of sending sensor data to the onenet server. Mobile device through the WiFi to visit the main control board Ardiunomage2560 sensor data, Using HMI USART display sensor data, if the environmental parameters exceed the set value, using the GPRS GSM module to send text messages to inform the user,the whole 
system is simple, convenient and fast, to achieve the desired objectives.

\section{B. Main Control Board and All Kinds of Environmental Sensors}

Table 1.ArduinoIO ports and sensor connection table

\begin{tabular}{cc}
\hline Arduino Mage2560 io ports & Sensors and modules \\
\hline Uart1 & GSM GPRS Module \\
Uart2 & ESP8266-12F \\
Uart3 & Dust Sensor \\
Uart4 & Usart HMI \\
A0 & Sound Sensor \\
A1 & CO $_{2}$ Sensor \\
A2 & Gas Sensor(MQ2) \\
A3 & Flame Sensor \\
A4 & Wind speedSensor \\
A5 & WinddirectionSensor \\
D7 & SoilTemperatureAnd Humidity \\
D8 & Sensor(SHT10) \\
D1 & Air TemperatureAnd Humidity \\
& Sensor(DHT21) \\
IIC & BarometerSensor(BMP10) \\
D9 D10 D11 D12 & Light Sensor(BH1750FVI) \\
\hline
\end{tabular}

\section{OneNET China Mobile IoT Open Platform}

OneNETis built by China Mobile IoT Open Platform, which responds"mass entrepreneurship and innovation". OneNET is beased on promotion of massive connection,cloud computing, light application and big data,to construct a vision of IoT ecosystem.OneNET platform provides massive connections, cloud stroage, message distribution, reverse control, capability output, application incubation and other quality services.OneNET faces vast number of enterprises, makers and students in IOT industry, to satisfy IOT requirement of quick connection, data storage, data security. Collect the environmental parameters sent to ONENET display platform.[6]

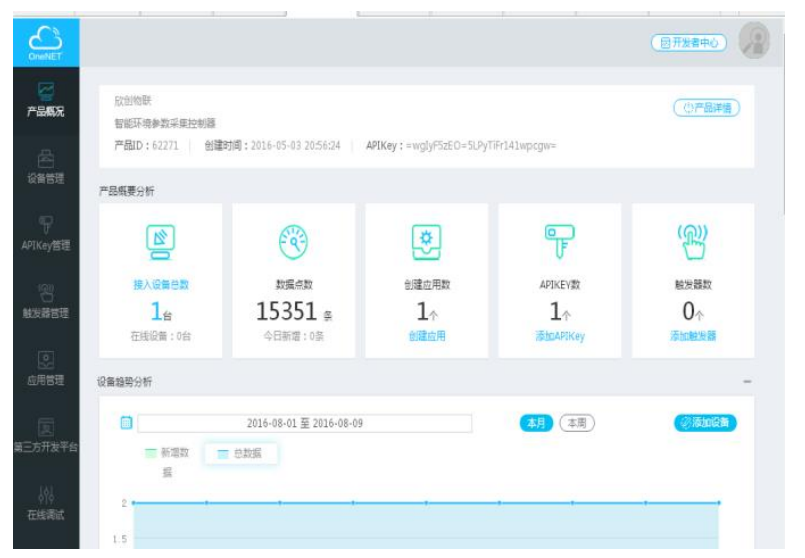

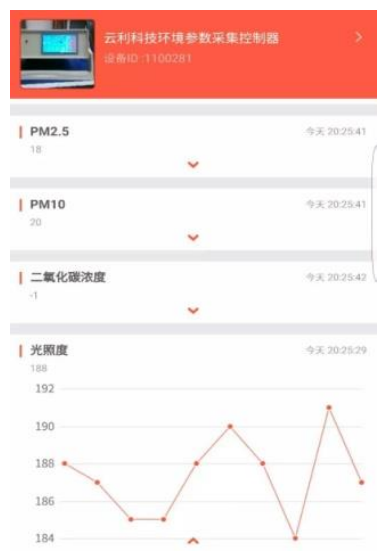

Figure 3. OneNET China Mobile IoT Open Platform

\section{The System SofTWARE DESIGN}

Arduino Programming language similar to $\mathrm{C} / \mathrm{C}++$, because Arduino group pre in the integrated software development environment provides commonly used library file, with its basic function only they can call, these basis functions including I / O control, time function, learning function, trigonometric function and so on. This design highlights the low threshold and hardware independent, so that developers quickly get started, do not take into account the underlying hardware implementation, and focus on the application itself.[7][8][9]

In order to realize the network transmission function, we have adopted the ESP8266 WiFi Module[3][5][6],Key code to send sensor data to the onenet platform.

/************************************************ $* * * * * * * * * * * * * * * * * * * * * * * * *$

post toOneNet

String API_VALUE

unsigned long device_id

String sensor_id int thisData

*************************************************

$* * * * * * * * * * * * * * * * * * * * * * * * * /$

void

API_VALUE,unsigned sensor id,int thisData)

if(newMux(TCP,OneNetServer,80)) \{

DBGLN("connecting...");

// send the HTTP PUT request: String cmd;

cmd $=$

"POST

http://api.heclouds.com/devices/";

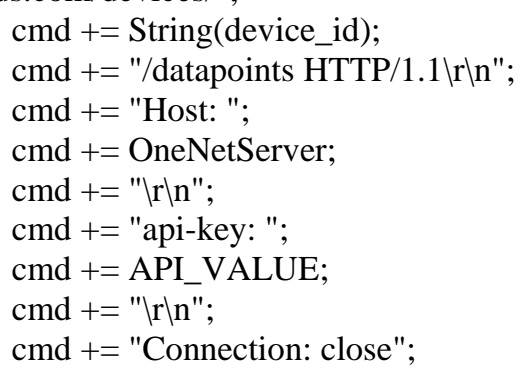


getLength(thisData);

cmd += "\r|n";

cmd += "Content-Length: ";

int length $=53+$ sensor_id.length ()$+$

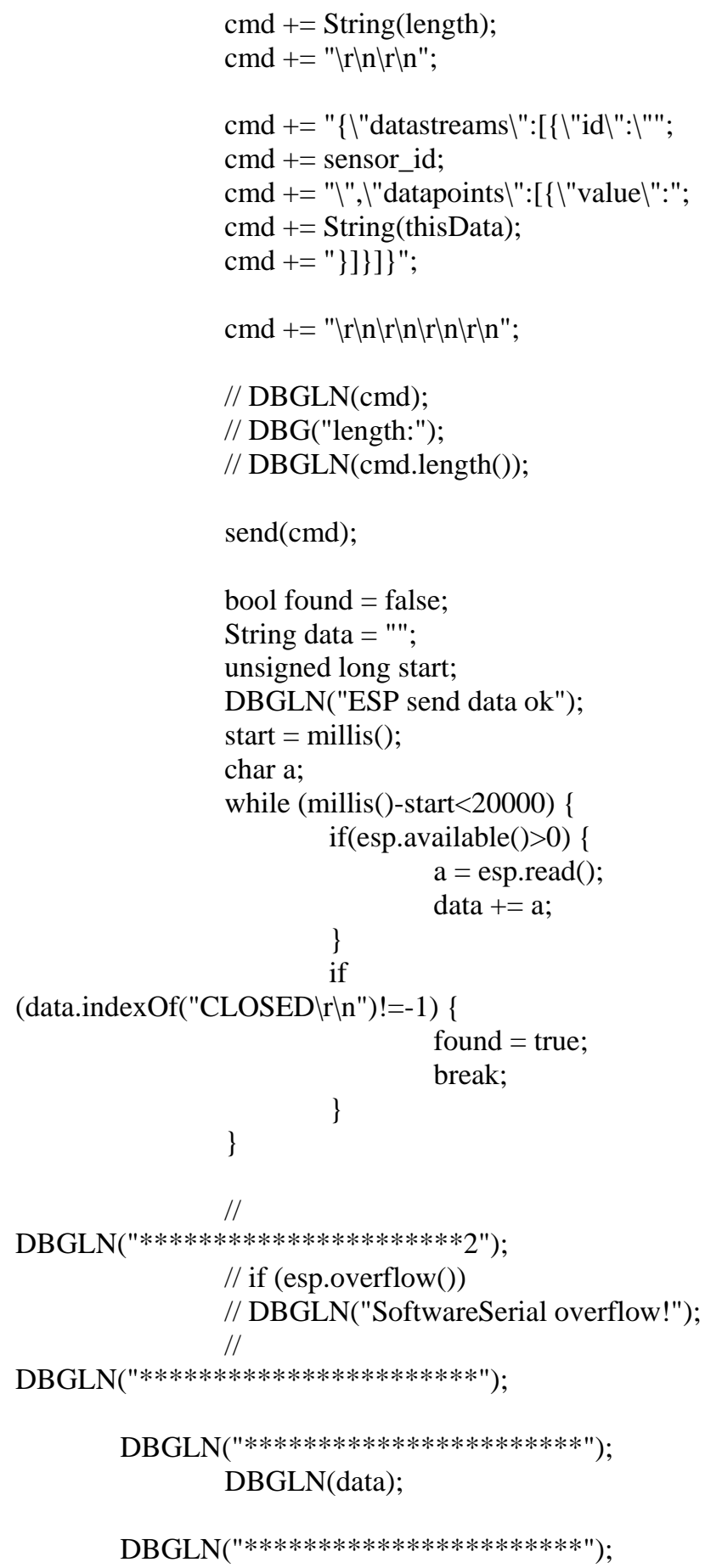

DBGLN(data.length());

$\operatorname{DBGLN}(" * * * * * * * * * * * * * * * * * * * * * * *))$

if(found)

DBGLN("RECEIVE

।"CLOSED \"");

else \{

।"CLOSED।"!");

DBGLN("NOT

RECEIVE

\} else \{

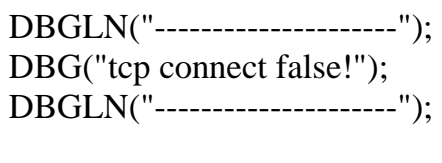

\}

So far, to send data to the onenet platform to achieve functional.

\section{Conclusion}

This paper takes arduinomage 2560 as the core, combined with various types of sensors, touch screen, WiFi module, GSM GPRS module,design and implementation of a IDC environmental monitoring controller,With onenet as the Internet of things cloud platform.the research results of this paper can be widely cited in the data center room environmental parameters monitoring, can also be applied to intelligent agriculture, lake water quality monitoring, personal weather stations, environmental pollution monitoring and other fields.

\section{References}

[1] https://www.arduino.cc/

[2] http://www.instructables.com/.

[3] Josh Adams.Beginning Arduino. 2010

[4] Massimo Banzi.Getting Started with Arduino. 2008.

[5] http://www.seeedstudio.com/wiki/WiFi_Serial_Transceiver_Modu le.

[6] http://open.iot.10086.cn/doc/art/id/200\#56.

[7] Cai Ruiyan. The Principle and Application of Arduino [J]. Electronic Design Engineering. 2012 (16).

[8] Tao Ye. Play the arduino iot application [M]. Beijing: Beijing University of Aeronautics and Astronautics Press, 2013

[9] Li Yonghua. Arduino Hardware and Software Co - Design Guide [M]. Beijing: Tsinghua University Press, 2015

[10] Zhao Zi. Arduino Development Guide [M]. Beijing: Mechanical Industry Press, 2015 